\title{
Analytical Method Development for Determination of Performance of Adapalene in Adapalene 0.1\% Gel Formulation Using Manual Diffusion Cell
}

\author{
S.S.DEO, F. INAM and N. P. KARMARKAR ${ }^{*}$ \\ Department of Chemistry, Institute of Science, \\ Rabindranath Tagore Road, Civil Lines, Nagpur-440010, India \\ nitinpkarmarkar@rediffmail.com
}

Received 4 August 2012 / Accepted 4 September 2012

\begin{abstract}
A simple, reliable and reproducible method applicable for topical creams is developed to monitor the in vitro performance of adapalene $0.1 \%$ cream formulations using manual diffusion cell with suitable synthetic membrane and receptor medium. The membranes which have permeability similar to skin and receptor media that can maintain the sink conditions have been selected. For solid oral dosage forms such as tablets, extensive study and analytical methods were developed to evaluate the in vitro performance of the drug. But for semisolid dosage forms, no official guideline exists to measure its performance. Therefore, to quantify the performance of the adapalene $0.1 \%$ cream, in vitro release method is developed with manual diffusion cell and analysed using high performance liquid chromatography.
\end{abstract}

Keywords: In vitro release, Manual diffusion cell, Synthetic support membranes, Receptor medium, Reverse phase-high performance liquid chromatography

\section{Introduction}

When the drug is applied topically, its active pharmaceutical ingredient released from the carrier or vehicle before it can come in contact with the epidermis surface and be available for the penetration in the Stratum corneum and the lower layer of the skin. The topical formulation is a complex system and the dynamics of the release of the drug has been the subject of investigation from many years. The in vitro diffusion test of semisolid dosage forms is an official requirement for the pharmaceutical industries to determine the drug availability and to ensure the batch to batch uniformity. The developments of such methods help to establish the bioequivalence of the product after Scale Up and Post Approval Changes (SUPAC). The development of the such methods for the determination of in vitro release of the topical dermatological formulations is applicable for the different types of topical dosage forms.

The development of in vitro release study serves as a good quality control tool to ensure batch to batch uniformity and screen experimental formulation during the product development. Determination of the value of in vitro release helps to cross check the product 
quality and product comparison. As a thumb rule, the percentage of the drug not more than $30 \%$ of the API, should be released in the donor compartment of manual diffusion cell ${ }^{1}$. The various stages of development of analytical method to study the in vitro release of adapalene $0.1 \% \mathrm{w} / \mathrm{w}$ cream formulation is discussed in this article. The essential considerations for the development of in vitro study are discussed as follows.

\section{Characteristics of synthetic membranes}

Synthetic membranes have been extensively used to determine the in vitro release characteristics of an API from topical formulations ${ }^{2-7}$. These membranes are not intended to mimic the barrier properties or the heterogeneous nature of the $\operatorname{skin}^{8}$. Instead, they are designed to provide a physical support and maintain constant contact between the formulation and receptor medium and to prevent bulk transfer of the dosage form, while allowing the API to release from a formulation matrix into a receptor medium. Membranes are not required for rigid formulations such as ointment. However, creams and gels invariably contain an aqueous phase and adjuvant components that are aqueous in nature and are water-miscible. Therefore, a membrane must be placed between the formulation being tested and the receptor medium in order to maintain the physical integrity of individual formulations or dosage forms ${ }^{9}$. Synthetic membranes that have been commonly used for in vitro release studies are those with porous characteristics such as cellulose acetate $\mathrm{e}^{10-12}$, nitrocellulose ${ }^{13}$ and polycarbonate membrane filters ${ }^{14,15}$ or alternatively homogeneous permeable polymers such as silicone based filter membranes ${ }^{16}$.

An important consideration for selecting synthetic membrane for use in vitro drug release experiments is its commercial availability. In addition, the membrane should have low reactivity with formulation components, be compatible with the receptor medium and offer the least possible diffusional resistance to the component of interest ${ }^{17-18}$. The membrane of choice should be inert and provide a holding surface without barrier properties for the active ingredient and test formulation ${ }^{19}$.

The migration of a drug from a semi-solid matrix into a receptor medium is essentially a function of one or a combination various processes such as drug release from the semi-solid matrix itself, passage of the drug through the membrane and clearance of the drug from below the membrane. Therefore, it is important that the membrane and the receptor medium be highly permeable and accessible to the drug in the formulation for efficient drug release. By considering above aspects regarding the synthetic membrane, different synthetic membranes such as nylon 66, PVDF, cellulose, acetate, supor and tuffryn of pore size $0.45 \mu$ were used for in vitro release study of $0.1 \% \mathrm{w} / \mathrm{w}$ adapalene formulation.

\section{Characteristics of receptor medium}

While selecting receptor medium for in vitro release test, it is necessary that medium has adequate solubility of API throughout the course of an experiment without impacting on the sink conditions of the system. Ideally, receptor media should be aqueous and isotonic solutions. However, for products formulated with water-insoluble drugs, the selection of an appropriate receptor medium to maintain sink characteristics is a challenge. In order to facilitate and monitor drug release from such topical formulations, it is necessary to alter $\mathrm{pH}$ of receptor medium, addition of surfactants such as sodium lauryl sulphonate and complexing agents or use non-aqueous media in which the drug is more soluble and efficiently released from the matrix during in vitro studies. 
For the present study, tetrahydrofuran (THF), various concentration of sodium lauryl sulphate, tween 60 and tween 80 buffers, phosphate buffer and hydro-ethanolic medium were used to study the in vitro release of the adapalene $0.1 \%$ gel.

\section{Experimental}

The various adapalene formulations of $0.1 \% \mathrm{w} / \mathrm{w}$ were selected from market. The Differin gel containing adapalene gel $0.1 \% \mathrm{w} / \mathrm{w}$ was used as a reference innovator sample for the comparison of in vitro release of other formulations. In vitro release of samples was performed on manual diffusion cell and analysed on reverse phase-high performance liquid chromatography. The cumulative amount release (Q) of adapalene per surface area of the membrane was calculated using the following equation ${ }^{20}$.

$$
\mathrm{Q}=\mathrm{CnV}+\sum_{\downarrow}(i=1)^{\uparrow}(n-1[\{(C i s)\} / A])
$$

Where, Q represents cumulative amount of adapalene released per sq. $\mathrm{cm}$ of the membrane $\left(\mu \mathrm{g} / \mathrm{cm}^{2}\right)$, Cn represents concentration of adapalene $(\mu \mathrm{g} / \mathrm{mL})$ determined at the $\mathrm{n}^{\text {th }}$ sampling interval, $\mathrm{V}$ represents volume of individual manual diffusion cell, $\sum_{i=1}^{n-1}$ (Cis) represents sum of concentrations of adapalene $(\mu \mathrm{g} / \mathrm{mL})$ determined at sampling intervals 1 through n-1, S represents volume of sampling aliquot, A represents surface area of sampling well.

\section{Diffusion parameters}

After evaluating different receptor media, synthetic membranes diffusion method parameters have been developed. Receptor medium was prepared by mixing 65 volumes of absolute alcohol ethanol with 35 volumes of HPLC water and sonicated for 10 mins. The sample cell temperature of manual diffusion cell and receptor medium container was maintained at $32 \pm 0.5$ ${ }^{\circ} \mathrm{C}$ using temperature controller. Prior to the start of experiment, receptor media was filled in six diffusion cell for temperature equilibration. The magnetic stirrer helix of each diffusion cell helps to keep the receptor medium stirring continuously at $400 \mathrm{rpm}$. The speed of rotation was controlled through the speed controller.

\section{Sample application and diffusion cell preparation}

The previously soaked membrane in isopropyl myristate was attached to the dosage wafer from the lower side. Approximately $300 \mathrm{mg}$ (equivalent maximum fill) of cream was filled in the central hole of the dosage wafer. This contains $0.3 \%$ of adapalene API and as per in vitro release test condition (IVRT), release of adapalene from formulation should not be more than $90 \mu \mathrm{g} / \mathrm{cm}^{2}$. The sample was spread uniformly on the dosage wafer with the help of Teflon spreader. The entrapment of air bubble occluded between membrane and receptor medium was avoided during sample application. This membrane along with sample was kept in contact with the receptor medium. The dosage wafer was covered with glass disk to prevent solvent losses due to evaporation from the cream. Membrane, dosage wafer and glass disk was kept in alignment with help of alignment ring. This ring was used to align membrane and glass disk and also used to fit these components compactly. All the above components such as alignment ring, glass disk and membrane were fitted using an aluminium clamp. All the remaining six manual diffusion cells were prepared in similar way. The internal standard solution of $40 \mathrm{mg} \mathrm{mL}^{-1}$ of adapalene API was prepared considering highest release of the cream in receptor medium. Samples were taken at an interval of $0.0,1.0,2.0,4.0,6.0$ and 8.0 hours and analysed along with internal standard using 
RP-HPLC method. The required quantity of sample for analysis was $2.0 \mathrm{~mL}$. The sample was collected from sample port in HPLC vial by pushing the fresh medium through replace port using the calibrated syringe. Different market samples were studied and analysed on RP-HPLC.

\section{Chromatographic parameters}

The samples that have been taken at different time intervals were analysed by liquid chromatographic method. Chromatographic separation was performed on Waters high performance liquid chromatography with 2695 separation module and 2487 dual $\lambda$ absorbance detector system. Chromatographic separation was performed using Phenomenex, Luna RP-150 $\mathrm{mm} \times 4.6 \mathrm{~mm} \times 5 \mu, 100-3$, column and mobile phase comprising a mixture triflouroacetic acid buffer $(0.1 \%)$ and acetonitrile $(20: 80) \mathrm{v} / \mathrm{v}$, at a flow rate of $1.0 \mathrm{~mL} \mathrm{~min}^{-1}$ and UV detection at $225 \mathrm{~nm}$. Samples were injected in volumes of $50 \mu \mathrm{L}$. Peak purity of drug was checked using photo diode array detector. Chromatograms and data were recorded by using Empower software.

\section{Results and Discussion}

During the experiments, it was observed that tuffryn membrane when used along with the hydroalcoholic medium shows a very good in vitro release pattern as compared with the remaining membranes and hydroalcoholic medium. Hydro ethanolic medium (65\%) shows a good solubility and tuffryn membrane provide a good permeability for adapalene to release in the receptor medium. The increasing concentrations of sodium lauryl sulphonate (SLS) and tween 60, tween 80 buffers show a remarkable drop in the release over the period of experiment. This is due to the blocking of pores of the membranes due to the heavier molecules of surfactants over the period of time restricting the API to diffuse in the receptor medium. To study the effect of $\mathrm{pH}$ on release pattern, aqueous phosphate buffers of $\mathrm{pH} 3.5$ and $\mathrm{pH} 5.5$ were used. However, no remarkable effect on release pattern has been observed. Thus, $30 \%$ in vitro release condition of adapalene $0.1 \%$ formulation can be obtained using hydro-ethanolic medium and tuffryn membrane. Chromatographic separation pattern for internal standard and adapalene gel $0.1 \%$ from Bionova pharmaceuticals is shown in the Figure 1 and Figure 2 respectively. All the formulations have similar chromatographic patterns. The Figure 1 and Figure 2 shows the chromatographic pattern obtained which is very simple and can be applied to the variety of formulations as indicated in Table 2. The retention time of adapalene peak is around 8.250 minutes and total run time is 10 minutes. Thus, the time required for analysis is very less.

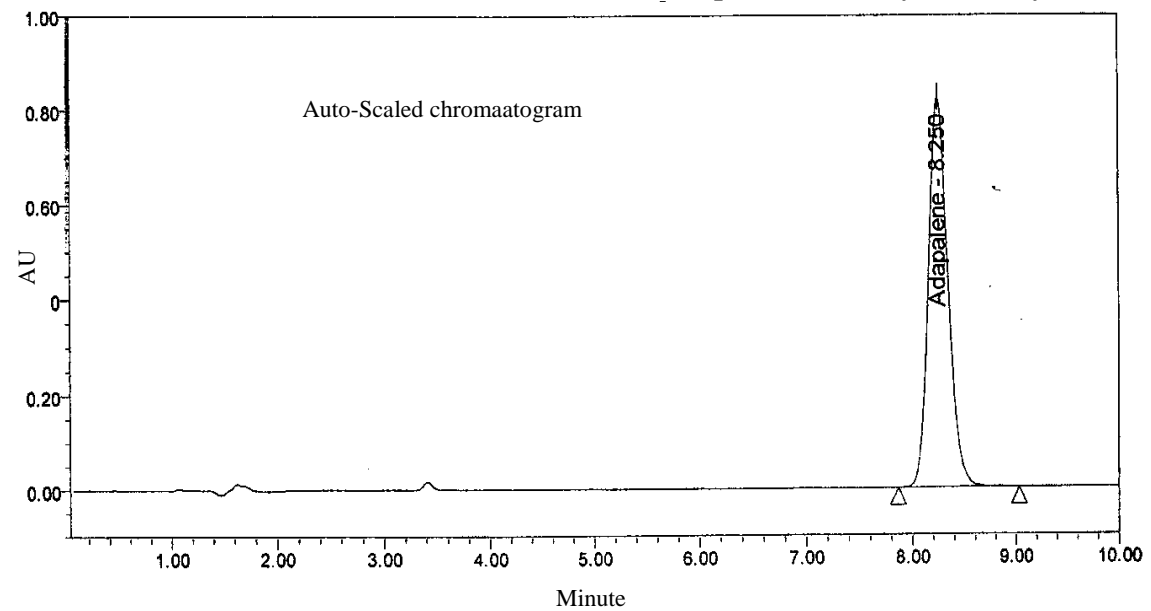

Figure 1. Chromatographic pattern for adapalene internal standard 


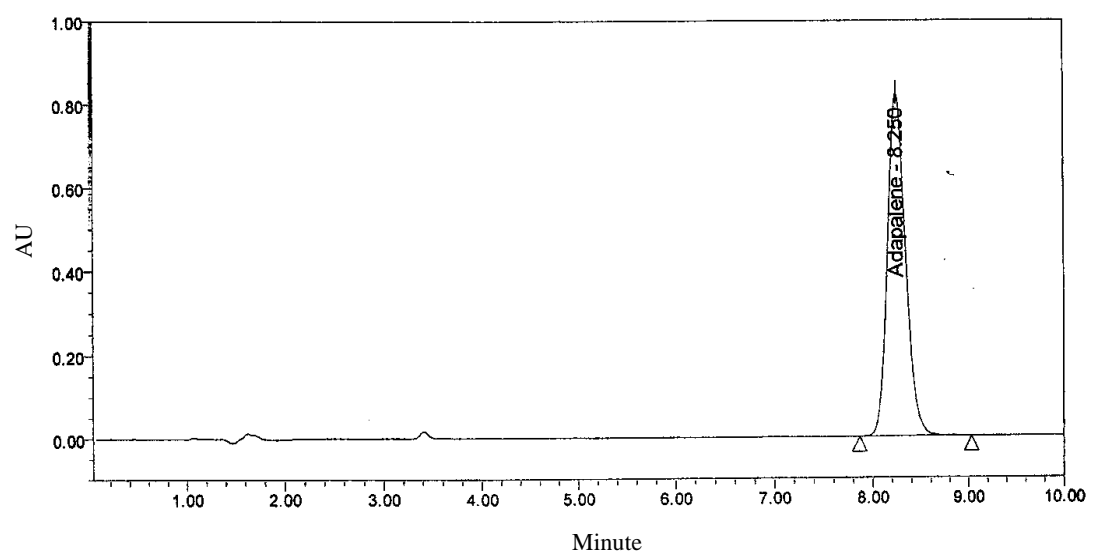

Figure 2. Chromatographic pattern for adapalene gel $0.1 \%$

The in vitro release (in $\mu \mathrm{g} / \mathrm{cm}^{2}$ ) of adapalene gel $0.1 \%$ from Bionova pharmaceuticals in different receptor media and using tuffryn membrane is shown in Table 1 . The in vitro release (in $\mu \mathrm{g} / \mathrm{cm}^{2}$ ) of all the market formulations used in experiment is shown in the Table 2.

Table 1. In vitro release (in $\mu \mathrm{g} / \mathrm{cm}^{2}$ ) of adapalene (bionova) formulation in different media using tuffryn membrane

\begin{tabular}{ccccccccc}
\hline S.No & $\begin{array}{c}\text { Sq. rt. of } \\
\text { time, mins }\end{array}$ & THF & $\begin{array}{c}0.5 \% \\
\text { SLS }\end{array}$ & $\begin{array}{c}2.00 \% \\
\text { SLS }\end{array}$ & $\begin{array}{c}\text { pH 3.5 } \\
\text { Buffer }\end{array}$ & $\begin{array}{c}\text { pH } \\
\text { Buffer }\end{array}$ & $\begin{array}{c}\text { Tween 80 } \\
\text { Buffer }\end{array}$ & $\begin{array}{c}\text { Alcohol } \\
\text { (65\%) }\end{array}$ \\
\hline 1 & 0 & 0 & 0 & 0 & 0 & 0 & 0 & 0 \\
2 & 7.74 & 10.24 & 9.56 & 6.55 & 15.55 & 16.59 & 5.99 & 5.99 \\
3 & 10.95 & 14.23 & 13.24 & 9.98 & 21.45 & 22.56 & 11.24 & 8.24 \\
4 & 15.49 & 28.23 & 22.36 & 19.25 & 29.56 & 32.15 & 13.24 & 35.24 \\
5 & 18.97 & 51.23 & 33.25 & 29.35 & 43.26 & 46.29 & 13.98 & 56.98 \\
6 & 21.90 & 70.18 & 45.78 & 42.15 & 52.86 & 63.59 & 14.02 & 92.02 \\
\hline
\end{tabular}

Where,pH 3.5 buffer-aqueous 5\% Phosphate buffer pH 3.5 (65:35). pH 5.5 buffer-aqueous 5\% Phosphate buffer $p H 5.5$ (65:35), THF=Tetrahydrofuran, $S L S=$ Sodium lauryl sulphonate

Table 2. The in vitro release (in $\mu \mathrm{g} / \mathrm{cm}^{2}$ ) of all the marketed samples

\begin{tabular}{ccccc}
\hline $\begin{array}{c}\text { S. } \\
\text { No }\end{array}$ & Formulations & Brand Name & Company & $\begin{array}{c}\mu \mathrm{g} / \mathrm{cm}^{2} \\
\text { release }\end{array}$ \\
\hline 1 & Adapelene 0.1\% gel & Diffryn gel & Galderma & 92.56 \\
2 & $\begin{array}{c}\text { Adapelene 0.1\% + Clindamycin } \\
\text { Phophate Gel 1.0\% }\end{array}$ & Adacin & Ajanta & 93.21 \\
3 & Adapelene gel 0.1\% & Adiff Aq.gel & Bionova & 90.12 \\
4 & Adapelene gel 0.1\% & Adapelene gel 0.1\% & Talent India & 91.58 \\
5 & Adapelene gel 0.1\% & Adaferine 0.1\% & Galderma & 91.98 \\
\hline
\end{tabular}

\section{Conclusion}

In order to study the solid oral dosage forms extensive study has been developed, but for semisolid dosage forms no official guideline has been given. Only few quality control tests, such as the determination of solubility, particle size and size distribution and crystalline form of an API and evaluation of the intrinsic viscosity and homogeneity of a final product has been traditionally used to provide reasonable evidence of consistent product manufacture and performance. Therefore, it was very difficult to predict the sameness and 
consistent performance of the product. Hence, an effort was made to develop a method that can be used in conjunction with the traditional quality control tools. The result of the present study supports the usage of currently developed method as a quality control tool to estimate the product performance and sameness. A very simple and reliable method has been developed for the in vitro release of the adapalene $0.1 \%$ cream formulation. The in vitro release provides the good evidence for product evaluation and performance. The method can be easily reproduced. The use of five different formulations ensures that the method developed helps to differentiate between the formulations of different manufacturers. The current diffusion method can sense the smaller changes occurred during the scale up and post approval changes. Thus, the method justifies its use as a quality control tool and enables the manufacturer to adjust the different types of formulations. Method is used to determine the quality and consistency of adapalene topical cream formulations that are to be manufactured during product development studies. The in vitro release method was also used to characterize formulations and evaluate batch-to-batch consistency and assess the impact of storage at elevated temperatures on product performance. Moreover, after the development of diffusion method, method is validated using the standard method validation protocol which justifies the ruggedness and reproducibility of the method.

RP-HPLC method developed is a powerful and reliable analytical tool that can be used for the in vitro analysis of a formulation which is of a complex nature such as adapalene cream/gel formulations, gels and ointments. During the chromatographic development studies, all the required HPLC parameters are validated as per the standard USP protocol. Therefore, the developed chromatographic method has good control over the variation of the product qualities. The chromatographic method HPLC not only provides separation and quantitative data but also has the ability to eliminate almost all interference challenges.

\section{References}

1 Shah V P, Elkins J S, and Williams R L, Pharm Dev Techno1., 1999, 4, 377-385.

2 Shah V P, Elkins J, Hanus J, Noorizadeh C and Skelly J P, Int J Pharm.,1989, 53(1), 53-59.

3 Corbo M, Shultz T W, Wong G K and Van Buskirk G A, Pharm Technol., 1993 , 17(9), 112-128.

4 Shah V P, Elkins J, Lam S Y and Skelly J P, Int J Pharm., 1989, 53(1), 53-59.

$5 \quad$ Shah V P, Elkins J and Williams R L, Pharm Develop Technol., 1999, 4(3), 377-385.

6 Dias M, Farinha A, Faustino E, Hadgraft J, Pais J and Toscano C, Int J Pharm., 1999, 182(1), 41-47.

$7 \quad$ Shah V P and Elkins J S, J Pharm Sci., 1995, 84(9), 1139-1140.

8 Zatz J L and Segers J D, Dissolution Tech., 1998, 5(1), 3-13.

9 Flynn G L, Shah V P, Tenjarla S N, Corbo M, DeMagistris D, Feldman T G, Franz T J, Miran D R, Pearce D M, Sequeira J A, Swarbrick J, Wang J C, Yacobi A and Zatz J L, Pharm Res., 1999, 16(9), 1325-1330.

10 Chattaraj S C and Kanfer I, Int J Pharm., 1996, 133(1-2), 59-63.

11 Liebenberg W, Engelbrecht E, Wessels A, Evarakonda B, Yang W and De Villiers M M, J Food Drug Anal., 2004,12(1), 19-28.

12 Dias M, Farinha A, Faustino E, Hadgraft J, Pais J, Toscano C, Int J Pharm.,1999, 182(1), 41.

13 Klimundova J, Satinsky D, Sklenarova H and Solich P, Talanta, 2006, 69(3), 730-735.

14 Klimundova J, Sklenarova H, Schaefer U F and Solich P, J Pharm Biomed Anal., 2005, 37(5), 893-898. 
15 Csoka I, Csanyi E, Zapantis G, Nagy E, Feher-Kiss A and Horvath G, Blazsó G and Eros I, Int J Pharm., 2005, 291(1-2), 11-19.

16 Pefile S C, Smith E W, Albrecht C F and Kruger P B, Int J Pharm., 1998, 161(2), 237-243.

17 Zatz L J, Pharm Res., 1995, 12(5), 787-789.

18 Shah V P, Elkins J, Shaw S and Hanson R, Pharm Dev Technol., 2003, 8(1), 97-102.

19 Thakker K D and Chern W H, Dissolution Technol., 2003, 10(5), 10-15.

20 a) Thakker K and Chern W H, Dissolution Technol., 2001, 125-129; b) Shah V P, Stevan W S, Norton D D, Elkins J and Hanson R, Pharm Forum, 2006, 32(5), 712. 